\title{
Acquisition of gemcitabine resistance enhances angiogenesis via upregulation of IL-8 production in pancreatic cancer
}

\author{
HIROYUKI IMAFUJI*, YOICHI MATSUO*, GORO UEDA, KAN OMI, YUICHI HAYASHI, \\ KENTA SAITO, KEN TSUBOI, MAMORU MORIMOTO, SHUJI KOIDE, RYO OGAWA, \\ MASAYASU HARA, HIROKI TAKAHASHI and SHUJI TAKIGUCHI
}

Department of Gastroenterological Surgery, Nagoya City University Graduate School of Medical Sciences, Mizuho-cho, Mizuhoku, Nagoya, Aichi 467-8601, Japan

Received September 18, 2018; Accepted March 20, 2019

DOI: $10.3892 / o r .2019 .7105$

\begin{abstract}
Gemcitabine (Gem) is widely used as chemotherapy for pancreatic cancer $(\mathrm{PaCa})$, but its effect is not fully satisfactory. One of the reasons for this is the acquisition of Gem resistance (Gem-R). To elucidate the mechanism of Gem-R, two Gem-R PaCa cell lines were established from AsPC-1 and MIA PaCa- 2 cells. It was demonstrated that expression of interleukin-8 (IL-8) mRNA was significantly upregulated in Gem-R PaCa cells by cDNA microarray and RT-qPCR analyses. Increased IL-8 secretion by Gem-R cells was confirmed by cytokine array and enzyme-linked immunosorbent assay. Moreover, we found that co-culture with Gem-R PaCa cells significantly enhanced tube formation of human umbilical vein endothelial cells, and treatment with an anti-CXCR2 (main receptor for IL-8) antibody significantly prevented this effect. We previously reported that a chemokine network centered on the IL-8/CXCR2 axis plays an important role in PaCa angiogenesis, and suppression of this axis has an antitumor effect. Since acquisition of Gem-R increased IL- 8 production and consequently increased tumor angiogenesis, the IL-8/CXCR2
\end{abstract}

Correspondence to: Dr Yoichi Matsuo, Department of Gastroenterological Surgery, Nagoya City University Graduate School of Medical Science, Kawasumi 1, Mizuho-cho, Mizuhoku, Nagoya, Aichi 467-8601, Japan

E-mail: matsuo@med.nagoya-cu.ac.jp

${ }^{*}$ Contributed equally

Abbreviations: Gem, gemcitabine; $\mathrm{PaCa}$, pancreatic cancer; Gem-R, gemcitabine resistance; Gem-S, gemcitabine sensitive; IL-8, interleukin-8; CXCR2, C-X-C motif chemokine receptor 2; FBS, fetal bovine serum; $\mathrm{IC}_{50}$, half maximal inhibitory concentration; RT-qPCR, reverse transcription-quantitative polymerase chain reaction; ELISA, enzyme-linked immunosorbent assay; HUVECs, human umbilical vein endothelial cells; Ab, antibody; SD, standard deviation

Key words: IL-8, gemcitabine, gemcitabine resistance, pancreatic cancer, CXCR2, angiogenesis, chemokine axis may be a potential novel therapeutic target for $\mathrm{PaCa}$ after acquiring Gem-R.

\section{Introduction}

Pancreatic cancer $(\mathrm{PaCa})$ is one of the most highly malignant carcinomas. $\mathrm{PaCa}$ was the fourth leading cause of cancer-related death in Japan in 2017 (National Cancer Center Japan. Center for Cancer Control and Information Services, 2018. Cancer Statistics in Japan. https://ganjoho. jp/en/ professional/statistics/table_download.html.) and the third leading cause of cancer-related death in the US. In 2018, there were an estimated 55,440 newly diagnosed patients with $\mathrm{PaCa}$ and 44,330 PaCa-related deaths in the US (1). The overall 5 -year survival rate is $\sim 6 \%$ (range, $2-9 \%$ ) worldwide (2). The poor prognosis of $\mathrm{PaCa}$ is attributed to its typical characteristics of late presentation, aggressive local invasion, early metastasis, and poor response to chemotherapy (3). Most $\mathrm{PaCa}$ patients are already at an advanced stage at the time of diagnosis. Approximately $10-20 \%$ of $\mathrm{PaCa}$ patients are eligible for tumor resection, whereas the remaining patients are eligible only for adjuvant therapies (4-6). Therefore, the development of novel target therapies is expected to improve the outcome of $\mathrm{PaCa}$ treatment.

In 1997, a phase III trial comparing fluorouracil (5-FU) monotherapy, which was the standard treatment at that time, and gemcitabine (Gem) monotherapy for patients with $\mathrm{PaCa}$ was conducted. That trial demonstrated the advantages of Gem over 5-FU not only in terms of a clinical benefit (e.g., pain relief) but also overall survival (5.7 vs. 4.4 months) (7). Consequently, Gem was approved in 1997 as a first-line chemotherapeutic drug for patients with locally advanced or metastatic $\mathrm{PaCa}$, and $\mathrm{Gem}$ remains the standard treatment for PaCa patients (4). However, the clinical efficacy of Gem therapy is poor, and there is little improvement in the survival of $\mathrm{PaCa}$ patients who receive this therapy (6). Clinical experience has shown that Gem has transient effects on $\mathrm{PaCa}$ after beginning chemotherapy, with the effects readily decreasing thereafter. One of the reasons is acquisition of Gem resistance (Gem-R), the mechanism of which is still unclear. Thus, it is reasonable to expect that elucidation of Gem-R mechanisms will improve $\mathrm{PaCa}$ treatment. 
Recently, there has been increasing evidence that chemokines play a role in tumor biology $(8,9)$. These studies have demonstrated that chemokines may influence tumor growth, invasion and metastasis. Interleukin-8 (IL-8/CXCL8) is a CXC chemokine containing a Glu-Leu-Arg motif and is secreted by leukocytes and tumor cells. It was initially named neutrophil-activating peptide-1 for its potent chemotactic activity on granulocytes in inflammatory and immune diseases (10). IL-8 has been shown to play important roles in cancer invasion (11), angiogenesis (12) and metastasis (13). Based on these results, previous reports indicate that IL- 8 is a potential therapeutic target in breast $(14,15)$, gastric $(13,16)$, colon $(17)$, cervical cancer (18), and melanoma (19). Furthermore, we previously demonstrated a significant role of PaCa-induced IL-8 in tumor angiogenesis (20,21). Moreover, blocking CXCR2, the main receptor of IL-8, significantly suppressed the increased $\mathrm{PaCa}$ angiogenesis and tumorigenesis (20).

The aim of this study was to determine the role of IL- 8 in the acquisition of Gem- $\mathrm{R}$ in PaCa. Initially, we established Gem-R PaCa cell lines. We confirmed that IL-8 expression was increased in accordance with the acquisition of Gem-R. Furthermore, we found that an anti-CXCR2 Ab inhibited the angiogenic activity induced by Gem- $\mathrm{R}$ in $\mathrm{PaCa}$ cell lines. To the best of our knowledge, this is the first report to demonstrate the mechanisms of Gem-R-induced tumor angiogenesis in relationship to the chemokine network. Since regulation of the IL-8/CXCR2 axis reduced Gem-R-induced angiogenesis, this axis may be a new therapeutic target for Gem- $\mathrm{R} \mathrm{PaCa}$.

\section{Materials and methods}

Cell culture and treatments. The PaCa cell lines BxPC-3, AsPC-1, MIA PaCa-2, Panc-1, and SW 1990 were obtained from the American Type Culture Collection (ATCC; Rockville, MD, USA). BxPC-3 and AsPC-1 cells were maintained in RPMI-1640 medium (Sigma Chemical Co.; Merck KGaA, Darmstadt, Germany) supplemented with $10 \%$ fetal bovine serum (FBS). MIA PaCa-2, Panc-1 and SW 1990 cells were maintained in Dulbecco's modified Eagle's medium (DMEM) (Sigma Chemical Co.; Merck KGaA) containing high glucose and $10 \% \mathrm{FBS}$. All cells were incubated at $37^{\circ} \mathrm{C}$ in a humidified atmosphere of $5 \% \mathrm{CO}_{2}$ in air.

Establishment of Gem-R PaCa cell lines. First, we determined the half maximal inhibitory concentration $\left(\mathrm{IC}_{50}\right)$ of Gem (Toronto Research Chemicals, North York, Ontario, Canada) for PaCa cells using the Premix WST-1 Cell Proliferation Assay System (Takara Bio, Inc., Shiga, Japan) according to the manufacturer's instructions. Briefly, PaCa cells (AsPC-1, MIA PaCa-2, BxPC-3, Panc-1 and SW 1990) were seeded at $2 \times 10^{3} / 100 \mu \mathrm{l}$ in 96 -well plates and allowed to adhere overnight. Then, the cultures were provided with fresh medium containing various concentrations of Gem. After $72 \mathrm{~h}$ of incubation, the absorbance was measured at $450 \mathrm{~nm}$ in each well using the SpectraMax 340 spectrophotometer (Molecular Devices, LLC, Sunnyvale, CA, USA). The $\mathrm{IC}_{50}$ of Gem for each PaCa cell line was determined by constructing dose-response curves. Each $\mathrm{PaCa}$ cell line was passaged at the respective $\mathrm{Gem} \mathrm{IC}_{50}$ concentration for 2-3 weeks. After passage, the $\mathrm{IC}_{50}$ value of Gem was again determined for each cell line and each cell line was passaged with the re-determined $\mathrm{IC}_{50}$ concentration for 2-3 weeks. The process was repeated at increasing doses of Gem until the cell lines demonstrated at least a 50-fold greater Gem $\mathrm{IC}_{50}$ value than that of the respective parental cell line. The resulting cell lines were resistant to $20 \mu \mathrm{M}$ Gem.

Cell proliferation assay. Proliferation assays were conducted using the Premix WST-1 Cell Proliferation Assay System (Takara Bio, Inc.) according to the manufacturer's instructions. Briefly, Gem-R and Gem-sensitive (Gem-S) AsPC-1 or MIA PaCa- 2 cells were seeded at $2 \times 10^{3} / 100 \mu 1$ in 96 -well plates and allowed to adhere overnight. Then, cultures were provided with fresh medium containing various concentrations $(0-1,000 \mu \mathrm{M})$ of Gem. After $72 \mathrm{~h}$ of incubation, the absorbance was measured at $450 \mathrm{~nm}$ in each well using the SpectraMax 340 spectrophotometer (Molecular Devices, LLC). To compare the proliferation of Gem-R and Gem-S $\mathrm{PaCa}$ cells in the absence of Gem treatment, $2 \times 10^{4}$ cells were seeded in 6-well plates and allowed to adhere overnight. After $24,48,72$ and $96 \mathrm{~h}$ of incubation, the cells were isolated after treatment with $0.25 \%$ Trypsin-EDTA (Gibco; Thermo Fisher Scientific, Inc., Waltham, MA, USA) and counted in four microscopic fields (x100) using a compound light microscope. This assay was also performed to investigate the effect of IL-8 on the proliferation of Gem-R and Gem-S PaCa cells. First, Gem-S MIA PaCa-2 cells were incubated with various concentrations $(0-100 \mu \mathrm{M})$ of Gem and with or without $100 \mathrm{ng} / \mathrm{ml}$ recombinant human CXCL8/IL-8 (R\&D Systems, Minneapolis, MN, USA) for $72 \mathrm{~h}$. Second, Gem-R and Gem-S $\mathrm{PaCa}$ cells were treated with IL-8 small-interfering RNA (siRNA) or negative control siRNA (as described below) to assess the effect of siRNA-mediated knockdown of IL-8. In both cases, the cells were subjected to WST-1 proliferation assays as described above.

siRNA-mediatedIL-8knockdown. We performed siRNA-mediated knockdown of IL- 8 in Gem-R PaCa cells using IL-8 siRNA(h) (cat. no. sc-39631; Santa Cruz Biotechnology, Inc., Dallas, TX, USA), Silencer Select Negative Control No. 1 siRNA (4390843) (Invitrogen; Thermo Fisher Scientific, Inc.), Opti-MEM I reduced serum medium (Invitrogen; Thermo Fisher Scientific, Inc.), and Lipofectamine ${ }^{\circledR}$ RNAiMAX transfection reagent (Invitrogen; Thermo Fisher Scientific, Inc.) according to the manufacturers' instructions. Briefly, Gem-R MIA PaCa-2 cells were seeded in 6-well plates and allowed to adhere overnight. Then, the cells were incubated with transfection mixtures containing $10 \mathrm{nM}$ IL-8 siRNA or negative control siRNA for $72 \mathrm{~h}$. IL-8 expression was confirmed in $\mathrm{PaCa}$ cells treated with IL-8 siRNA or negative control siRNA by reverse-transcription quantitative polymerase chain reaction (RT-qPCR) as described below. The IL-8 siRNA (cat. no. sc-39631) oligonucleotides were 5'-GGGUGCAGA GGGUUGUGGAGAtt-3' (sense), and 5'-UCUCCACAACCC UCUGCACCCtt-3' (antisense).

Total mRNA microarray analysis. Total mRNA from Gem-R and Gem-S MIA PaCa-2 cells were isolated using the RNeasy Plus Mini Kit (Qiagen, Inc., Valencia, CA, USA) according to the manufacturer's instructions. The mRNA microarray experiments were performed at the Takara Bio Dragon Genomics 
Center (Yokkaichi, Mie, Japan). Transcripts amplified from the total mRNA were hybridized to the Affymetrix Human Genome U133 Plus 2.0 Array (Affymetrix/Thermo Fisher Scientific, Inc.) according to the manufacturer's protocol. The results were analyzed using the Affymetrix GeneChip ${ }^{\mathrm{TM}}$ Command Console Software and Affymetrix Expression Console Software (Affymetrix; Thermo Fisher Scientific, Inc.).

$R T-q P C R$. Total RNA was isolated from PaCa cells using the RNeasy Plus Mini kit (Qiagen, Inc.), according to the manufacturer's instructions, and quantitated using the NanoDrop 1000 (Thermo Fisher Scientific, Inc.). Total RNA (1 $\mu \mathrm{g})$ was reverse-transcribed using the SuperScript III Platinum Two-Step qRT-PCR Kit (Invitrogen, Thermo Fisher Scientific, Inc.) according to the manufacturer's instructions, and $1 \mu \mathrm{l}$ of the product was used as a template for PCR. RT-qPCR was carried out using TaqMan Universal Master Mix and TaqMan Gene Expression Assays for IL-8 (Hs01553824_g1) and GAPDH (Hs99999905_m1) (Applied Biosystems; Thermo Fisher Scientific, Inc.) with Chrome4 (Bio-Rad, Hercules, CA, USA). The RT-qPCR conditions were as follows: an initial incubation at $50^{\circ} \mathrm{C}$ for $2 \mathrm{~min}$, followed by denaturation at $95^{\circ} \mathrm{C}$ for $10 \mathrm{~min}$ and 50 cycles of $95^{\circ} \mathrm{C}$ for $15 \mathrm{sec}$ and $60^{\circ} \mathrm{C}$ for $1 \mathrm{~min}$. The relative expression levels of IL- 8 were normalized to the expression of GAPDH in each sample using standard curve method.

Cytokine array. The Human Cytokine Array Panel Array kit (R\&D Systems) was utilized according to the manufacturer's instructions. Gem-R and Gem-S AsPC-1 cells were seeded at $1 \times 10^{6}$ in $60-\mathrm{mm}$ dishes and allowed to adhere overnight. Then, the culture medium was replaced with $1 \mathrm{ml}$ RPMI-1640 containing 2\% FBS. After $24 \mathrm{~h}$, the cell culture supernatant was centrifuged to remove particulates, and $500 \mu \mathrm{l}$ was used for each array. Images were captured using the LAS-3000 imaging system (Fujifilm, Shizuoka, Japan), with an exposure time of $10 \mathrm{~min}$. Signal analysis was performed using Multi Gauge software (ver3.0; Fujifilm, Tokyo, Japan), with each signal normalized to the positive controls.

Enzyme-linked immunosorbent assay (ELISA). All cell lines were seeded at $2 \times 10^{5} / \mathrm{ml}$ into a 24 -well plate containing medium supplemented with $10 \%$ FBS and cultured overnight. Medium was exchanged the next day, and cells were cultured for $48 \mathrm{~h}$. The culture media were then collected and centrifuged at $400 \mathrm{x}$ g for $5 \mathrm{~min}$ to remove particulates and frozen at $-80^{\circ} \mathrm{C}$ until used for ELISA. The concentration of IL-8 was measured using the Human CXCL8/IL-8 Quantikine ELISA kit (R\&D Systems) according to the manufacturer's instructions. The minimum detectable dose of this kit ranges from 1.5 to $7.5 \mathrm{pg} / \mathrm{ml}$.

Angiogenesis assay. To investigate the influence of Gem-R $\mathrm{PaCa}$ cells on tube formation by human umbilical vein endothelial cells (HUVECs), $\mathrm{PaCa}$ cell lines (Gem-R AsPC-1, Gem-S AsPC-1, Gem-R MIA PaCa-2, or Gem-S MIA PaCa-2), HUVECs and fibroblasts were co-cultured using a double chamber method in 24-well plates, and angiogenic activity was measured using an angiogenesis kit (cat. no. KZ-1000) (Kurabo Co., Osaka, Japan) according to the manufacturer's protocols (21-23). Gem-R or Gem-S $\mathrm{PaCa}$ cells $\left(1 \times 10^{4}\right.$ cells) were seeded into Transwell chambers, consisting of polycarbonate membranes with $0.45-\mu \mathrm{m}$ pores, and allowed to adhere overnight. Transwell chambers were then placed into the HUVEC/fibroblast co-culture system and exchanged on days 4, 7 and 10. Cells were cultured for 11 days, after which the HUVECs were stained with an anti-CD31 antibody (Ab) using a tubule staining kit (KZ-1225) (Kurabo Co.) according to the manufacturer's protocol. Briefly, we fixed cells by iced $70 \%$ ethanol for $30 \mathrm{~min}$ at room temperature. We used phosphate-buffered salts (-) with $1 \%$ bovine serum albumin as blocking reagent. We added mouse anti-human CD31 antibody in the kit diluted 4,000 times by blocking reagent as the primary $\mathrm{Ab}$ and incubated for $60 \mathrm{~min}$ at $37^{\circ} \mathrm{C}$. After incubation, we washed plates by blocking reagent and added goat anti-mouse IgG AlkP conjugate included in the kit diluted 500 times by blocking reagent as the secondary $\mathrm{Ab}$ and incubated for $60 \mathrm{~min}$ at $37^{\circ} \mathrm{C}$. The area of tube formation was measured quantitatively in 15 different fields for each condition using an image analyzer (Kurabo Co.). The assay allowed quantitative evaluation of angiogenesis and examination of tumor-stromal interactions. Using this same method, the effects of an anti-human neutralizing CXCR2 Ab $(10 \mu \mathrm{g} / \mathrm{ml}$; R\&D Systems) on HUVEC tube formation in the presence of $\mathrm{PaCa}$ cells were also assessed.

Statistical analysis. Differences between the means of two samples were analyzed by unpaired t-tests. Multiple group comparisons were performed by one-way analysis of variance (ANOVA) with a post hoc Bonferroni test for subsequent comparisons of individual groups. A P-value $<0.05$ was considered statistically significant. Mean values and standard deviations (SDs) were calculated for experiments performed in at least triplicate.

\section{Results}

$I C_{50}$ values of Gem for PaCa cell lines. To determine the concentrations of Gem needed to establish Gem-R PaCa cell lines, we performed cell proliferation assays. The $\mathrm{IC}_{50}$ of $\mathrm{Gem}$ for each $\mathrm{PaCa}$ line evaluated was determined by constructing dose-response curves (Fig. 1A). The $\mathrm{IC}_{50}$ values of Gem after $72 \mathrm{~h}$ of treatment were 10.4, 179.2, 122.5, 716.1 and $850.6 \mathrm{nM}$ for AsPC-1, BxPC-3, MIA PaCa-2, Panc-1 and SW 1990 cells, respectively (Fig. 1B).

Establishment of Gem-R PaCa cell lines. We incubated the five PaCa cell lines with $\mathrm{IC}_{50}$ values of Gem and successfully established two Gem-R PaCa cell lines from AsPC-1 and MIA PaCa- 2 cells. The $\mathrm{IC}_{50}$ values were 7,122.3 and $10.4 \mathrm{nM}$ for Gem-R and Gem-S AsPC-1 cells, respectively, and 12,614.3 and $122.5 \mathrm{nM}$ for Gem-R and Gem-S MIA PaCa-2 cells, respectively (Fig. 2). We investigated the biological differences between the Gem-S and Gem-R cell lines in subsequent experiments.

Proliferation of Gem-S and Gem-R PaCa cell lines. To compare cell proliferation between the Gem-R and Gem-S 


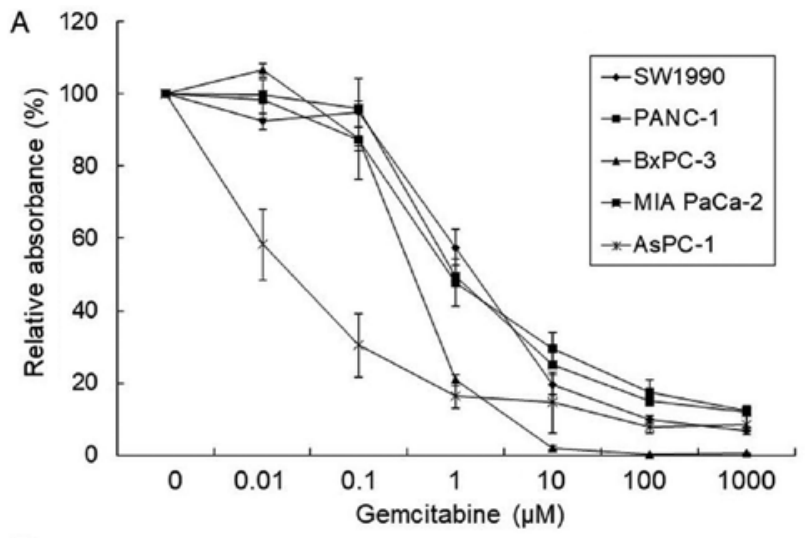

B

\begin{tabular}{cc}
\hline PaCa cell line & IC $_{50}$ for Gem \\
\hline AsPC-1 & $10.4 \mathrm{nM}$ \\
MIA PaCa-2 & $122.5 \mathrm{nM}$ \\
BxPC-3 & $179.2 \mathrm{nM}$ \\
Panc-1 & $716.1 \mathrm{nM}$ \\
SW 1990 & $860.6 \mathrm{nM}$ \\
\hline
\end{tabular}

Figure 1. Effect of Gem on the proliferation of PaCa cell lines. (A) Five PaCa cell lines (AsPC-1, MIA PaCa-2, BxPC-3, Panc-1 and SW 1990) were treated with Gem at the indicated concentrations for $72 \mathrm{~h}$, and the proliferation of each cell line was determined using WST-1 assays. Values are expressed as means $\pm \mathrm{SD}$. (B) The $\mathrm{IC}_{50}$ values of Gem for each $\mathrm{PaCa}$ cell line are shown. $\mathrm{Gem}$, gemcitabine; $\mathrm{PaCa}$, pancreatic cancer; $\mathrm{IC}_{50}$, half maximal inhibitory concentration.

PaCa cell lines, we performed proliferation assays. There was no significant difference in proliferation between the Gem-R and Gem-S cells of either line after 24-96 h of culture (Fig. 3).

cDNA microarray analysis of Gem-S and Gem-R MIA PaCa-2 cells. To investigate comprehensive differences in mRNA expression between Gem-S and Gem-R MIA PaCa-2 cells, we used a cDNA microarray containing 54,765 probe sets. Of these probes, 1,206 showed higher expression (cut-off value, 2-fold) and 3,157 lower expression (cut-off value, 0.5 -fold) in Gem-R compared with Gem-S MIA PaCa-2 cells (Table I). Among the genes with higher expression in the Gem-R cells, we focused on IL-8 as we previously reported an important role of IL-8 in $\mathrm{PaCa}$ angiogenesis (24).

RT-qPCR analysis of IL-8 mRNA expression in Gem-S and Gem-R PaCa cell lines. RT-qPCR revealed higher expression of IL-8 mRNA in Gem-R when compared with Gem-S cells in both $\mathrm{PaCa}$ cell lines evaluated $(\mathrm{P}<0.01$ in both AsPC- 1 cells and MIA PaCa-2 cells) (Fig. 4). The RT-qPCR results were consistent with the cDNA microarray results.

Upregulated IL-8 secretion by Gem-R compared with Gem-S $\mathrm{PaCa}$ cell lines. We examined differences in chemokine secretion by Gem-R and Gem-S AsPC-1 cells using cytokine arrays (Fig. 5A). The level of IL-8 was elevated in the culture supernatants of Gem-R compared with Gem-S cells, with signal intensity values of 0.44 and 0.32 , respectively (Fig. 5B).
Table I. cDNA microarray (54,675 genes).

Differentially expressed genes in Gem-R cells No. of genes

Upregulated genes (>2-fold) $\quad 1,206$

Downregulated genes $(<0.5$-fold $) \quad 3,157$

Gem-R, gemcitabine resistant.

A

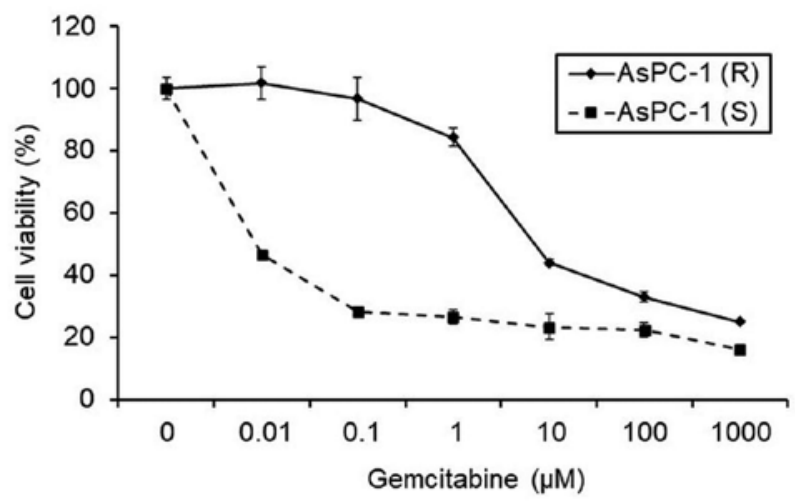

B

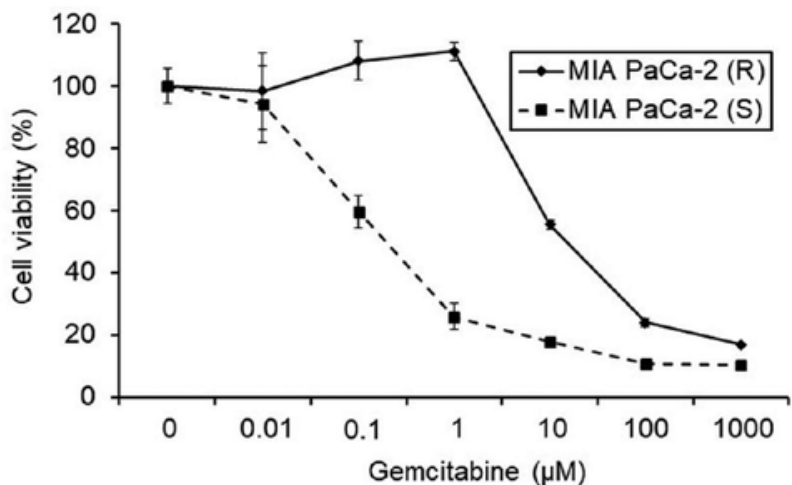

C

\begin{tabular}{cr}
\hline PaCa cell line & \multicolumn{1}{c}{$\mathrm{IC}_{50}$ for Gem } \\
\hline AsPC-1 (R) & $7,122.3 \mathrm{nM}$ \\
MIA PaCa-2 (R) & $12,614.3 \mathrm{nM}$ \\
\hline
\end{tabular}

Figure 2. Effect of Gem on the proliferation of Gem-R and Gem-S PaCa cell lines. (A and B) Gem-R and Gem-S AsPC-1 (A) and MIA PaCa-2 (B) cells were treated with Gem at the indicated concentrations for $72 \mathrm{~h}$, and the proliferation of each cell line was determined using WST-1 assays. Values are expressed as means $\pm \mathrm{SD}$. (C) The $\mathrm{IC}_{50}$ values of Gem in the Gem-R PaCa cell lines. AsPC-1 (R), Gem-R AsPC-1 cells; AsPC-1 (S), Gem-S AsPC-1 cells; MIA PaCa-2 (R), Gem-R MIA PaCa-2 cells; MIAPaCa-2 (S), Gem-S MIA PaCa-2 cells; Gem, gemcitabine; PaCa, pancreatic cancer; Gem-R, gemcitabine resistant; Gem-S, gemcitabine sensitive; $\mathrm{IC}_{50}$, half maximal inhibitory concentration.

We next examined IL-8 protein secretion by $\mathrm{PaCa}$ cell lines using ELISA (Fig. 5C). In both MIA PaCa-2 and AsPC-1 cell lines, IL-8 secretion was significantly enhanced in the Gem-R compared with the Gem-S cell lines $(\mathrm{P}<0.01$ for both cell lines). 

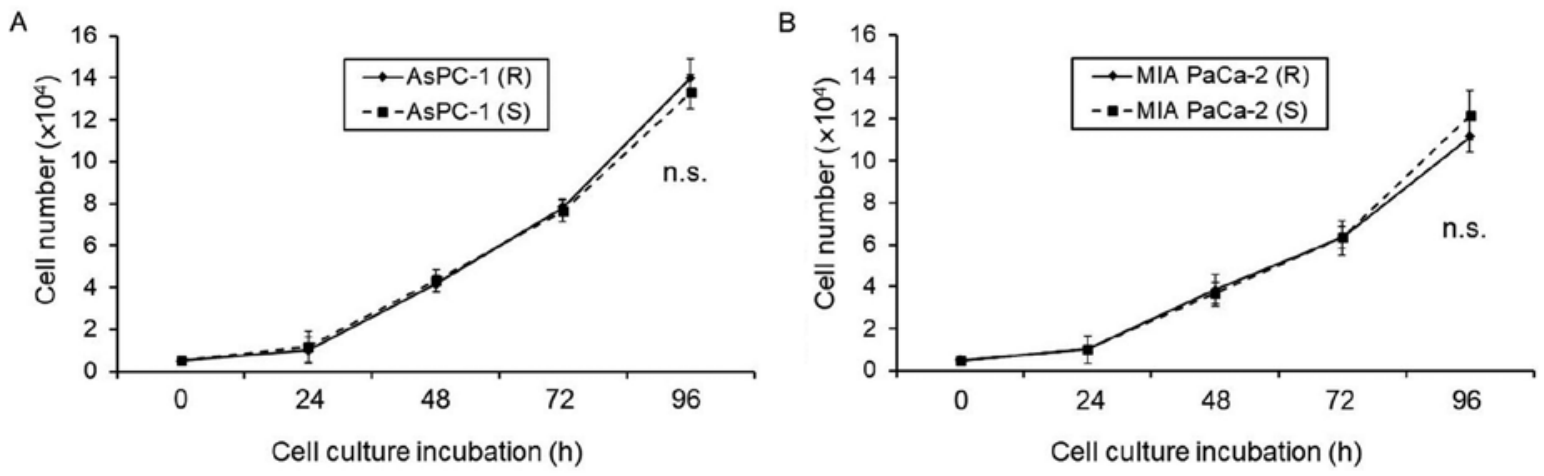

Figure 3. Differences in proliferation between Gem-R and Gem-S cells. (A and B) Gem-R and Gem-S AsPC-1 (A) and (B) MIA PaCa-2 (B) cells were seeded $\left(2 \times 10^{4}\right.$ cells) in 6-well plates and then collected after $0-96 \mathrm{~h}$ using $0.25 \%$ Trypsin-EDTA and counted in four microscopic fields (x100) using a compound light microscope. Values are expressed as means \pm SD. NS, not significant. There were no significant differences in cell numbers between the Gem-R and Gem-S cells of each cell line. AsPC-1 (R), Gem-R AsPC-1 cells; AsPC-1 (S), Gem-S AsPC-1 cells; MIA PaCa-2 (R), Gem-R MIA PaCa-2 cells; MIAPaCa-2 (S), Gem-S MIA PaCa-2 cells; Gem, gemcitabine; PaCa, pancreatic cancer; Gem-R, gemcitabine resistant; Gem-S, gemcitabine sensitive; $\mathrm{IC}_{50}$, half maximal inhibitory concentration; n.s., not significant.
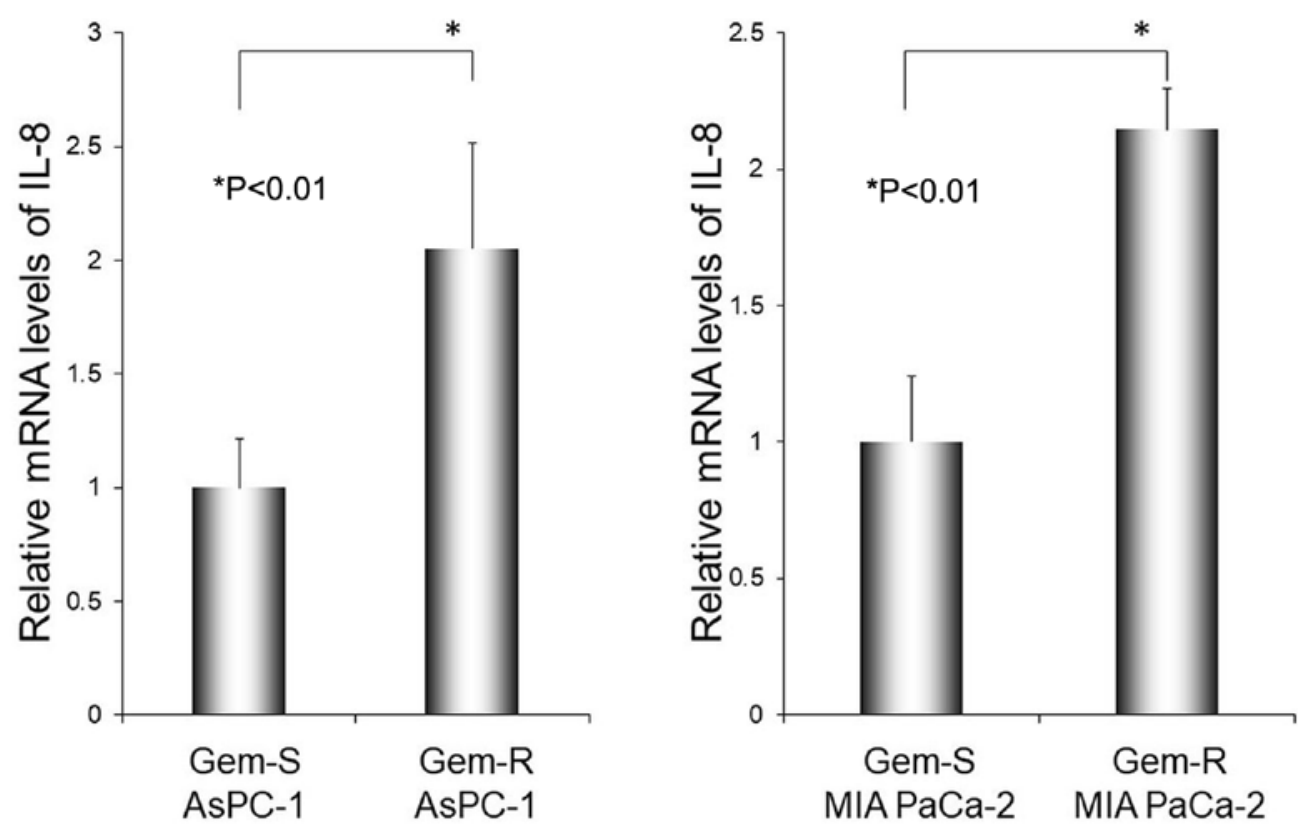

Figure 4. The IL-8 mRNA expression levels in Gem-R and Gem-S PaCa cell lines (AsPC-1 and MIA PaCa-2) were measured by RT-qPCR (normalized to GAPDH expression). Values are expressed as means \pm SD. Differences in means were analyzed by unpaired t-tests. ${ }^{*} \mathrm{P}<0.01$. Gem, gemcitabine; PaCa, pancreatic cancer; Gem-R, gemcitabine resistant; Gem-S, gemcitabine sensitive; IL-8, interleukin-8; RT-qPCR, reverse transcription-quantitative polymerase chain reaction.

Effect of IL-8 administration on the proliferation of PaCa cell lines. Gem-S PaCa cells were treated with or without IL-8 and various concentrations of Gem and then assessed by WST-1 proliferation assay. There was no significant difference in the proliferation of Gem-S MIA PaCa-2 cells treated with vs. not treated with IL-8 administration at any Gem concentration evaluated (Fig. S1).

Effect of siRNA-mediated IL-8 knockdown on the proliferation of Gem-R PaCa cells. RT-qPCR confirmed successful siRNA-mediated knockdown of IL-8 in Gem-R MIA PaCa-2 cells $(\mathrm{P}<0.01)$ (Fig. S2A). The effect of siRNA-mediated knockdown of IL-8 in Gem-R MIA PaCA-2 cells was assessed by WST-1 proliferation assays. There was no significant difference in the proliferation of Gem-R MIA PaCa-2 cells with vs. without IL-8 siRNA-mediated knockdown at any Gem concentration evaluated (Fig. S2B).

Effects of Gem-R development and an anti-CXCR2 Ab on HUVEC tube formation. To estimate the effect of Gem-R on angiogenesis, we performed an in vitro angiogenesis assay. HUVECs and fibroblasts were co-cultured with $\mathrm{PaCa}$ cell lines, and the effects of an anti-CXCR2 Ab were examined. Tube formation by HUVECs was significantly enhanced by co-culture with Gem-R compared with Gem-S $\mathrm{PaCa}$ cells ( $\mathrm{P}<0.01$ for both AsPC- 1 and MIA PaCa- 2 cells). Moreover, the enhanced tube formation by $\mathrm{PaCa}$ cells was inhibited by treatment with $(+)$ the anti-CXCR2 Ab $(\mathrm{P}<0.05$ 
A

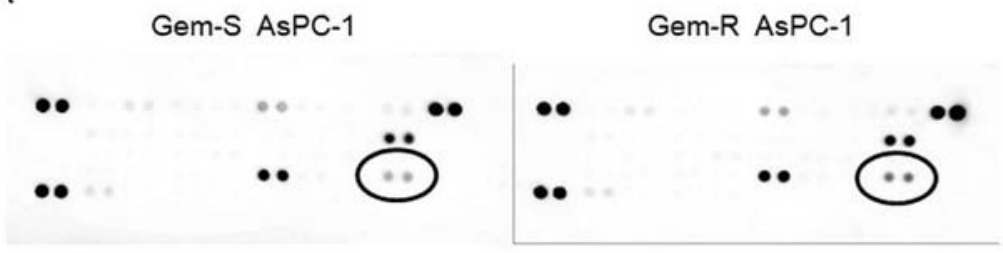

B

Gem-S AsPC-1

Gem-R AsPC-1

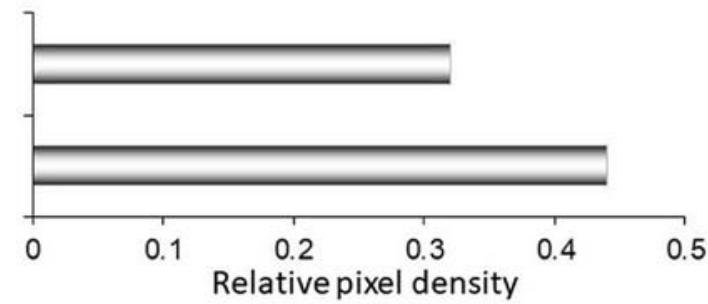

C
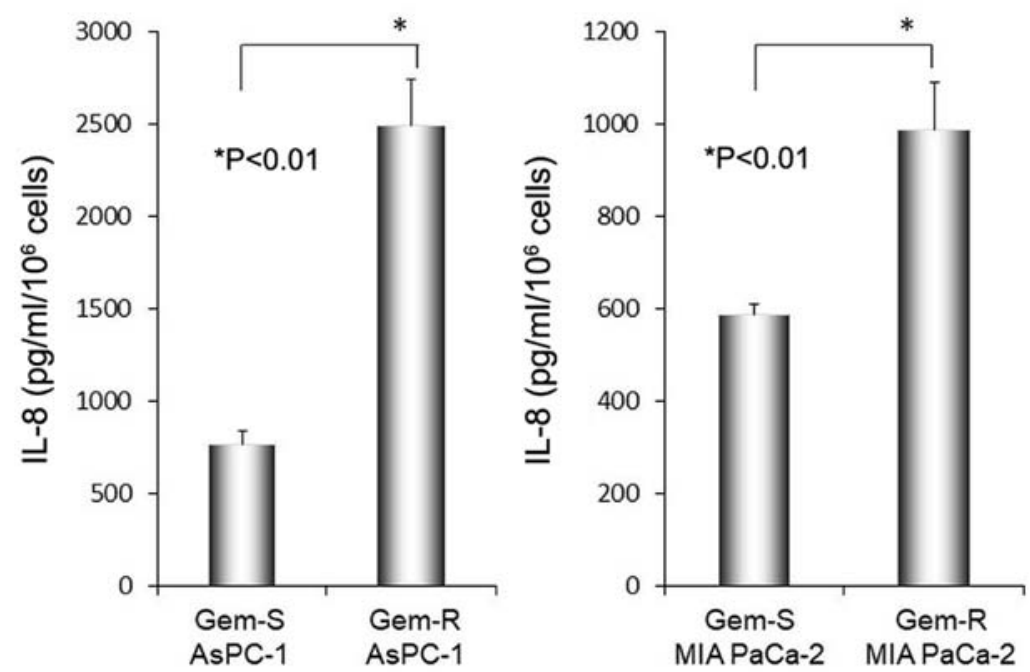

Figure 5. Differences in chemokine secretion between Gem-R and Gem-S PaCa cell lines. (A) Gem-R and Gem-S AsPC-1 cells were incubated for $24 \mathrm{~h}$ in RPMI-1640 supplemented with 2\% FBS. Secretion of chemokines from each cell line into the cell culture supernatant was measured by cytokine array. The circles indicate the spots representing IL-8. (B) The analyses of IL-8 expression signals from the cytokine array results were performed using Multi Gauge software, and each signal was normalized to that of the positive control. (C) Gem-R and Gem-S PaCa cell lines (AsPC-1 and MIA PaCa-2) were incubated for $48 \mathrm{~h}$, and the cell culture supernatants were collected. IL-8 protein levels in the cell culture supernatant were measured by ELISA. Values are expressed as means \pm SD. Differences in the means between two samples were analyzed by unpaired $t$-tests. " $\mathrm{P}<0.01$. Gem, gemcitabine; PaCa, pancreatic cancer; Gem-R, gemcitabine resistant; Gem-S, gemcitabine sensitive; IL-8, interleukin-8; ELISA, enzyme-linked immunosorbent assay.

for Gem-R MIA PaCa-2 cells and $\mathrm{P}<0.01$ for Gem-R and Gem-S AsPC-1 cells and Gem-R MIA PaCa-2 cells) (Fig. 6).

\section{Discussion}

In the present study, two main findings were confirmed. First, the resistance of pancreatic cancer $(\mathrm{PaCa})$ cells to gemcitabine $(\mathrm{Gem})$ was associated with increased interleukin (IL)-8 production from $\mathrm{PaCa}$ cells. Second, an anti-CXCR2 antibody (Ab), a neutralizing $\mathrm{Ab}$ for the main receptor of IL-8, inhibited the increase in angiogenesis induced upon acquisition of Gem resistance (Gem-R) in PaCa cells. Therefore, regulation of the IL-8/CXCR2 axis modulates the angiogenic activity and tumorgenicity of Gem-R PaCa cells.

Although chemotherapeutic strategies for $\mathrm{PaCa}$ are advancing, the therapeutic effects are still insufficient.
Gem-based chemotherapy is one of the standard treatments for patients with advanced PaCa. However, clinicians often find that Gem-based treatment usually has good initial effects, but that these effects often weaken shortly thereafter. One of the reasons for this is acquisition of Gem-R. To improve the effect of Gem chemotherapy on patient prognosis, a better understanding of the mechanism by which PaCa acquires Gem- $\mathrm{R}$ and development of new therapeutic strategies to overcome this resistance are required.

Previous studies have suggested several mechanisms of Gem-R. Gem-R can be acquired via molecular and cellular changes, including dysregulation of Gem metabolic pathways [e.g., deoxycytidine kinase and ribonucleotide reductase subunits M1 and M2 $(25,26)]$ and key signaling pathways [e.g., NF-кB (27,28), PI3K/Akt (28), MAPK, ERK1/2 (29), HIF-1 $\alpha$ (30)], increased expression of drug efflux pumps, 
A

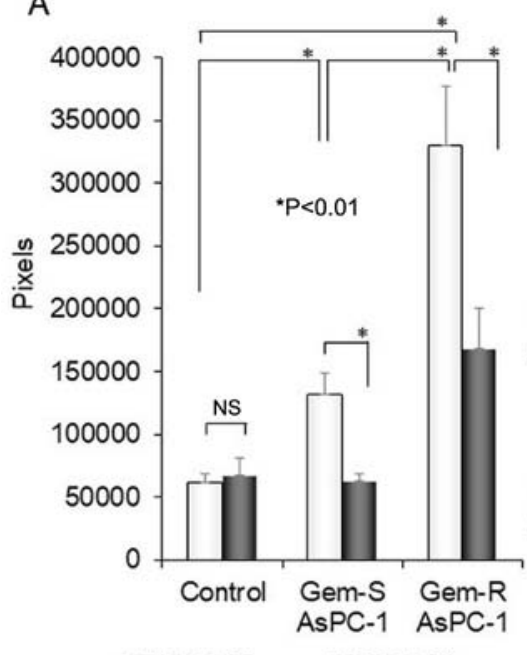

$\square C X C R 2 A b-\quad$ aCXCR2 Ab+

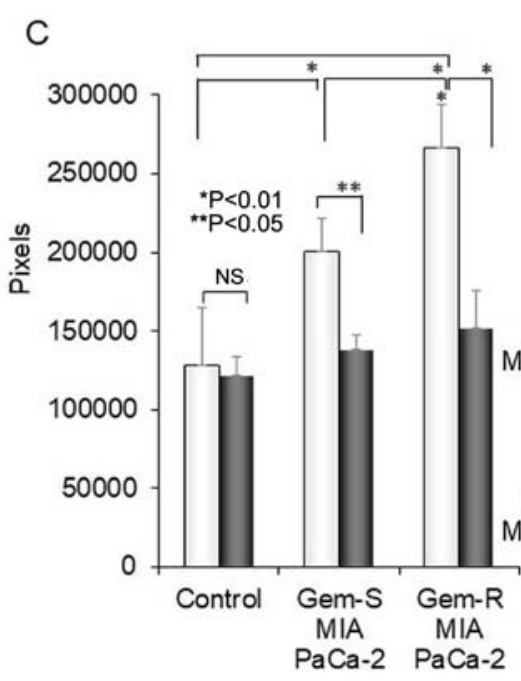

$\square C X C R 2 A b$ - $\because C X C R 2 A b+$
B
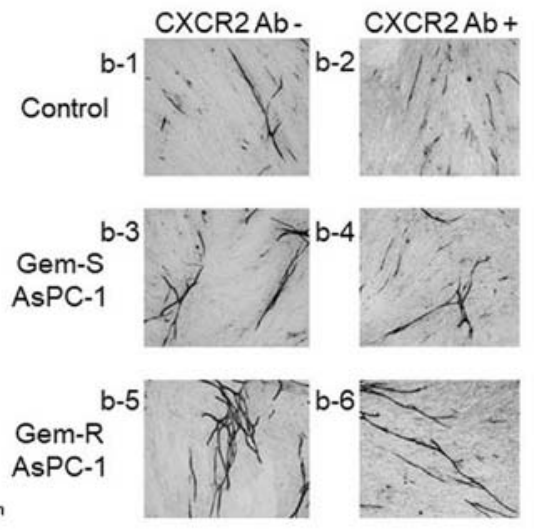

D
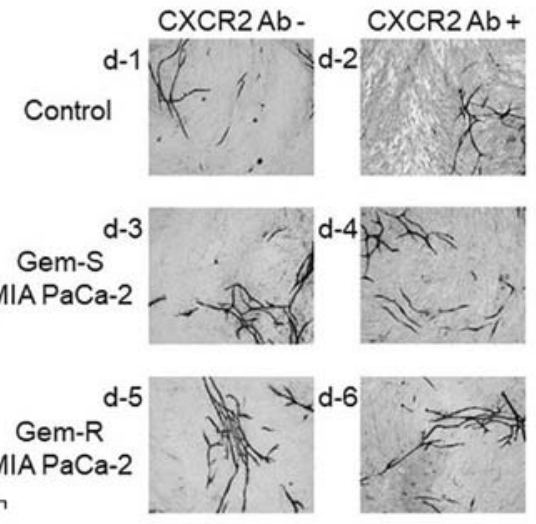

Figure 6. Differences in tube formation by HUVECs co-cultured with Gem-R and Gem-S PaCa cells and the effect of an anti-CXCR2 Ab. (A-D) After incubation of HUVECs, fibroblasts and the PaCa cell lines [Gem-R and Gem-S AsPC-1 cells (A and B) and MIA PaCa-2 cells (C and D)] for 11 days using the double-chamber method, HUVECs were stained with an anti-CD31 Ab. The tube formation area was measured using an image analyzer (magnification $\mathrm{x} 40$ ). Controls were co-cultured without PaCa cells. Values are expressed as means $\pm \mathrm{SD}$. Multiple group comparisons were performed by one-way analysis of variance (ANOVA) with a post hoc Bonferroni test. ${ }^{*} \mathrm{P}<0.01,{ }^{* *} \mathrm{P}<0.05$; NS, not significant. (B) (b-1) co-cultured without PaCa cells and CXCR2 Ab (-); (b-2) co-cultured without PaCa cells but with CXCR2 Ab (+); (b-3) co-cultured with Gem-S AsPC-1 cells but without CXCR2 Ab (-); (b-4) co-cultured with Gem-S AsPC-1 cells and CXCR2 Ab (+); (b-5) co-cultured with Gem-R AsPC-1 cells but without CXCR2 Ab (-); (b-6) co-cultured with Gem-R AsPC-1 cells and CXCR2 Ab (+). (D) (d-1) co-cultured without PaCa cells and CXCR2 Ab (-); (d-2) co-cultured without PaCa cells but with CXCR2 Ab (+); (d-3) co-cultured with Gem-S MIA PaCa-2 cells but without CXCR2 Ab (-); (d-4) co-cultured with Gem-S MIA PaCa-2 cells and CXCR2 Ab (+); (d-5) co-cultured with Gem-R MIA PaCa-2 cells but without CXCR2 Ab (-); (d-6) co-cultured with Gem-R MIA PaCa-2 cells and CXCR2 Ab (+). CXCR2 Ab -, not treated with CXCR2 antibody; CXCR2 Ab (+), treated with CXCR2 Antibody. Gem, gemcitabine; PaCa, pancreatic cancer; Gem-R, gemcitabine resistant; Gem-S, gemcitabine sensitive; CXCR2, C-X-C motif chemokine receptor 2; HUVECs, human umbilical vein endothelial cells; Ab, antibody. (B and D) Original magnification, x40.

activation of cancer stem cells (31), and epithelial-to-mesenchymal transition (32). However, a breakthrough clarifying the mechanism of Gem-R or identifying the appropriate secondary treatments have not been realized to date.

IL-8 is a pro-inflammatory factor belonging to the CXC chemokine family that is secreted by various cells to activate and recruit leukocytes to sites of infection and injury. Recently, several studies have reported that IL-8 plays important roles in cancer progression, angiogenesis and metastasis (33-35). Moreover, we previously demonstrated that IL- 8 is an important component of the tumor microenvironment in $\mathrm{PaCa}$, and that $\mathrm{PaCa}$ cells expressing high IL-8 levels have greater liver metastatic potential and angiogenic ability compared with $\mathrm{PaCa}$ cells expressing low IL-8 levels $(21,24)$. These results suggest that IL- 8 produced by $\mathrm{PaCa}$ cells regulates cancer progression and metastasis and may be an important therapeutic target. We also revealed that blockade of the IL-8/CXCR2 pathway significantly inhibited $\mathrm{PaCa}$ tumor growth by preventing angiogenesis, in vitro and in vivo (20). IL-8 activity occurs mainly via interaction with its specific cell-surface $G$ protein-coupled receptor, CXCR2 $(33,36,37)$. In our previous study, to inhibit the IL-8/CXCR2 pathway, we used both an anti-CXCR2 neutralizing $\mathrm{Ab}$ and a small-molecule inhibitor of CXCR2 (SB225002) (38); both had similar effects. 
A previous study showed that IL-8 is related to Gem-R and enhances the invasiveness of Gem-R PaCa (39). However, the specific role of IL-8 in Gem-R PaCa has not been sufficiently elucidated, particularly with respect to tumor angiogenesis. We reported that IL-8 plays an important role in tumor angiogenesis to affect $\mathrm{PaCa}$ metastasis and progression (40). In addition, we successfully established Gem-R PaCa cell lines and conducted comprehensive gene expression analyses. Among the altered genes, we found that the expression of IL-8, which was focused on in the present study, was increased in the Gem-R cells. Therefore, it is reasonable to expect that elucidating the mechanism of IL-8 involvement in the acquisition of Gem-R in $\mathrm{PaCa}$ will be important for the future development of $\mathrm{PaCa}$ treatments. Based on our previous and present research findings, IL-8 was selected as the focus of the present study.

CXCR2 is a cell-surface chemokine G protein-coupled receptor with seven transmembrane domains. CXCR2 has been found on the surfaces of endothelial, epithelial, and multiple tumor cells. Chemokines containing a Glu-Leu-Arg motif, such as CXCL1, CXCL2, CXCL3, CXCL5, CXCL6, and CXCL7 and IL-8, bind to CXCR2, and their interaction with CXCR2 tends to enhance tumor angiogenesis (37). High expression of CXCR2 showed an association with poor prognosis in laryngeal squamous cell carcinoma, lung cancer, clear-cell renal cell carcinoma, hepatocellular carcinoma, and pancreatic ductal carcinoma (41). There is abundant evidence on Gem-R and IL-8 $(39,42,43)$, but little is known concerning the effect of Gem-R on IL-8-induced angiogenesis in $\mathrm{PaCa}$. The mechanisms underlying how Gem- $\mathrm{R}$ acquisition induces IL-8 production in $\mathrm{PaCa}$ cells are not clear, but it was reported that Gem promotes NF- $\kappa \mathrm{B}$ activity in $\mathrm{PaCa}(42,44)$. As NF- $\kappa \mathrm{B}$ regulates IL-8 production (40), we speculate that IL-8 production in Gem- $\mathrm{R} \mathrm{PaCa}$ cells is associated with $\mathrm{NF}-\kappa \mathrm{B}$ activity, which is activated by Gem.

In the present study, to analyze the mechanism of Gem-R, we successfully established two Gem-R PaCa cell lines. Based on a comprehensive gene expression analysis, we found that the expression of IL-8 was elevated in the Gem-R PaCa cell lines. There was no significant change in proliferation between Gem-R and Gem-S cells. However, our in vitro angiogenesis assay indicated a significant increase in angiogenic activity in Gem-R cells. In this assay, we used a HUVEC/fibroblast co-culture system to assess the interactions between tumor cells and the tumor microenvironment and clearly demonstrated that an anti-CXCR2 Ab prevented the angiogenic activity induced by Gem-R. These results suggest that upregulation of IL-8 in Gem-R PaCa enhances angiogenesis via interaction with CXCR2, and blockade of the IL-8/CXCR2 signaling axis effectively reduces angiogenesis. These findings are consistent with previous studies that suggest that IL-8 expression is enhanced by Gem treatment in response to chemotherapy (42).

To determine whether the increase in IL- 8 production is the result vs. the initial cause of Gem-R acquisition, we examined the effect of suppression or overexpression of IL-8 on Gem-R. As neither recombinant IL-8 nor IL-8 siRNA treatment affected the proliferation of Gem-R cells compared with Gem-S cells, we believe that the increase in IL- 8 production may be the result, rather than the cause, of Gem-R.
In conclusion, we showed that acquisition of Gem-R increased IL-8 production. This increase in IL-8 production enhanced tumor angiogenesis in a co-culture environment consisting of $\mathrm{PaCa}$ and stromal cells. Importantly, we also demonstrated that blocking the IL-8/CXCR2 signaling axis using an anti-CXCR2 Ab affected Gem-R PaCa angiogenic activity. Based on these results, the IL-8/CXCR2 axis may be a potential novel therapeutic target for Gem- $\mathrm{R} \mathrm{PaCa}$.

\section{Acknowledgements}

Not applicable.

\section{Funding}

The present study was supported by the JSPS KAKENHI (grant no. 25462123).

\section{Availability of data and materials}

The datasets relevant to this study are included within the article.

\section{Authors' contributions}

Conception and design of the study were conducted by HI, YM and SK. Development of the study methodology was carried out by HI, YM, GU, KO, YH, KS, KT, MM, SK, RO, MH, HT and ST. Acquisition of the data was conducted by HI, YM and SK. Analysis and interpretation of the data were carried out by HI, YM and SK. Writing, review, and/or revision of the manuscript was carried out by HI, YM and SK. Writing of the manuscript in regards to the methods section by $\mathrm{GU}, \mathrm{KO}, \mathrm{YH}, \mathrm{KS}, \mathrm{KT}, \mathrm{MM}$, RO and HT. Administrative, technical, or material support was accomplished by YM and ST. Study supervision was carried out by YM. All authors read and approved the final manuscript and agree to be accountable for all aspects of the research in ensuring that the accuracy or integrity of any part of the work are appropriately investigated and resolved.

\section{Ethics approval and consent to participate}

Not applicable.

\section{Patient consent for publication}

Not applicable.

\section{Competing interests}

The authors declare that they have no competing interests.

\section{References}

1. Siegel RL, Miller KD and Jemal A: Cancer statistics, 2018. CA Cancer J Clin 68: 7-30, 2018.

2. Ilic M and Ilic I: Epidemiology of pancreatic cancer. World J Gastroenterol 22: 9694-9705, 2016.

3. Niedergethmann M, Alves F, Neff JK, Heidrich B, Aramin N, Li L, Pilarsky C, Grützmann R, Allgayer H, Post S, et al: Gene expression profiling of liver metastases and tumour invasion in pancreatic cancer using an orthotopic SCID mouse model. $\mathrm{Br}$ J Cancer 97: 1432-1440, 2007. 
4. Kamisawa T, Wood LD, Itoi T and Takaori K: Pancreatic cancer Lancet 388: 73-85, 2016.

5. Gillen S, Schuster T, Meyer Zum Büschenfelde C, Friess H and Kleeff J: Preoperative/neoadjuvant therapy in pancreatic cancer: A systematic review and meta-analysis of response and resection percentages. PLoS Med 7: e1000267, 2010.

6. Wong HH and Lemoine NR: Pancreatic cancer: Molecular pathogenesis and new therapeutic targets. Nat Rev Gastroenterol Hepatol 6: 412-422, 2009.

7. Burris HA III, Moore MJ, Andersen J, Green MR, Rothenberg ML, Modiano MR, Cripps MC, Portenoy RK, Storniolo AM, Tarassoff P, et al: Improvements in survival and clinical benefit with gemcitabine as first-line therapy for patients with advanced pancreas cancer: A randomized trial. J Clin Oncol 15: 2403-2413, 1997.

8. Raman D, Baugher PJ, Thu YM and Richmond A: Role of chemokines in tumor growth. Cancer Lett 256: 137-165, 2007.

9. Kulbe H, Levinson NR, Balkwill F and Wilson JL: The chemokine network in cancer-much more than directing cell movement. Int J Dev Biol 48: 489-496, 2004.

10. Baggiolini M, Walz A and Kunkel SL: Neutrophil-activating peptide-1/interleukin 8 , a novel cytokine that activates neutrophils. J Clin Invest 84: 1045-1049, 1989.

11. Kuwada Y, Sasaki T, Morinaka K, Kitadai Y, Mukaida N and Chayama K: Potential involvement of IL-8 and its receptors in the invasiveness of pancreatic cancer cells. Int J Oncol 22: 765-771, 2003.

12. Brat DJ, Bellail AC and Van Meir EG: The role of interleukin-8 and its receptors in gliomagenesis and tumoral angiogenesis. Neuro Oncol 7: 122-133, 2005.

13. Konno H, Ohta M, Baba M, Suzuki S and Nakamura S: The role of circulating IL-8 and VEGF protein in the progression of gastric cancer. Cancer Sci 94: 735-740, 2003.

14. Todorović-Raković N and Milovanović J: Interleukin-8 in breast cancer progression. J Interferon Cytokine Res 33: 563-570, 2013.

15. Lin Y, Huang R, Chen L, Li S, Shi Q, Jordan C and Huang RP: Identification of interleukin-8 as estrogen receptor-regulated factor involved in breast cancer invasion and angiogenesis by protein arrays. Int J Cancer 109: 507-515, 2004.

16. Shi J and Wei PK: Interleukin-8: A potent promoter of angiogenesis in gastric cancer. Oncol Lett 11: 1043-1050, 2016

17. Ning Y, Manegold PC, Hong YK, Zhang W, Pohl A, Lurje G, Winder T, Yang D, LaBonte MJ, Wilson PM, et al: Interleukin-8 is associated with proliferation, migration, angiogenesis and chemosensitivity in vitro and in vivo in colon cancer cell line models. Int J Cancer 128: 2038-2049, 2011.

18. Jia L, Li F, Shao M, Zhang W, Zhang C, Zhao X, Luan H, Qi Y, Zhang P, Liang L, et al: IL-8 is upregulated in cervical cancer tissues and is associated with the proliferation and migration of HeLa cervical cancer cells. Oncol Lett 15: 1350-1356, 2018.

19. Srivastava SK, Bhardwaj A, Arora S, Tyagi N, Singh AP, Carter JE, Scammell JG, Fodstad $\varnothing$ and Singh S: Interleukin- 8 is a key mediator of FKBP51-induced melanoma growth, angiogenesis and metastasis. Br J Cancer 112: 1772-1781, 2015.

20. Matsuo Y, Raimondo M, Woodward TA, Wallace MB, Gill KR Tong Z, Burdick MD, Yang Z, Strieter RM, Hoffman RM and Guha S: CXC-chemokine/CXCR2 biological axis promotes angiogenesis in vitro and in vivo in pancreatic cancer. Int J Cancer 125: 1027-1037, 2009.

21. Matsuo Y, Sawai H, Funahashi H, Takahashi H, Sakamoto M, Yamamoto M, Okada Y, Hayakawa T and Manabe T: Enhanced angiogenesis due to inflammatory cytokines from pancreatic cancer cell lines and relation to metastatic potential. Pancreas 28 : 344-352, 2004

22. Osugi T, Oshima Y, Fujio Y, Funamoto M, Yamashita A, Negoro S, Kunisada K, Izumi M, Nakaoka Y, Hirota H, et al: Cardiac-specific activation of signal transducer and activator of transcription 3 promotes vascular formation in the heart. J Biol Chem 277: 6676-6681, 2002.

23. Bishop ET, Bell GT, Bloor S, Broom IJ, Hendry NF and Wheatley DN: An in vitro model of angiogenesis: Basic features. Angiogenesis 3: 335-344, 1999.

24. Matsuo Y, Ochi N, Sawai H, Yasuda A, Takahashi H, Funahashi H, Takeyama H, Tong Z and Guha S: CXCL8/IL-8 and CXCL12/SDF-1alpha co-operatively promote invasiveness and angiogenesis in pancreatic cancer. Int J Cancer 124: 853-861, 2009.

25. Ohhashi S, Ohuchida K, Mizumoto K, Fujita H, Egami T, Yu J, Toma H, Sadatomi S, Nagai E and Tanaka M: Down-regulation of deoxycytidine kinase enhances acquired resistance to gemcitabine in pancreatic cancer. Anticancer Res 28: 2205-2212, 2008 .
26. Minami K, Shinsato Y, Yamamoto M, Takahashi H, Zhang S, Nishizawa Y, Tabata S, Ikeda R, Kawahara K, Tsujikawa K, et al: Ribonucleotide reductase is an effective target to overcome gemcitabine resistance in gemcitabine-resistant pancreatic cancer cells with dual resistant factors. J Pharmacol Sci 127: 319-325, 2015.

27. Waters JA, Matos J, Yip-Schneider M, Aguilar-Saavedra JR, Crean CD, Beane JD, Dumas RP, Suvannasankha A and Schmidt CM: Targeted nuclear factor-kappaB suppression enhances gemcitabine response in human pancreatic tumor cell line murine xenografts. Surgery 158: 881-889, 2015.

28. Arlt A, Gehrz A, Müerköster S, Vorndamm J, Kruse ML, Fölsch UR and Schäfer H: Role of NF-kappaB and Akt/PI3K in the resistance of pancreatic carcinoma cell lines against gemcitabine-induced cell death. Oncogene 22: 3243-3251, 2003.

29. Zheng C, Jiao X, Jiang Y and Sun S: ERK1/2 activity contributes to gemcitabine resistance in pancreatic cancer cells. J Int Med Res 41: 300-306, 2013

30. Wang R, Cheng L, Xia J, Wang Z, Wu Q and Wang Z: Gemcitabine resistance is associated with epithelial-mesenchymal transition and induction of HIF-1 $\alpha$ in pancreatic cancer cells. Curr Cancer Drug Targets 14: 407-417, 2014.

31. Chen M, Xue X, Wang F, An Y, Tang D, Xu Y, Wang H, Yuan Z, Gao W, Wei J, et al: Expression and promoter methylation analysis of ATP-binding cassette genes in pancreatic cancer. Oncol Rep 27: 265-269, 2012.

32. Quint K, Tonigold M, Di Fazio P, Montalbano R, Lingelbach S, Rückert F, Alinger B, Ocker M and Neureiter D: Pancreatic cancer cells surviving gemcitabine treatment express markers of stem cell differentiation and epithelial-mesenchymal transition. Int J Oncol 41: 2093-2102, 2012.

33. Liu Q, Li A, Tian Y, Wu JD, Liu Y, Li T, Chen Y, Han X and Wu K: The CXCL8-CXCR1/2 pathways in cancer. Cytokine Growth Factor Rev 31: 61-71, 2016

34. Li A, Dubey S, Varney ML, Dave BJ and Singh RK: IL-8 directly enhanced endothelial cell survival, proliferation, and matrix metalloproteinases production and regulated angiogenesis. J Immunol 170: 3369-3376, 2003.

35. Heidemann J, Ogawa H, Dwinell MB, Rafiee P, Maaser C, Gockel HR, Otterson MF, Ota DM, Lugering N, Domschke W and Binion DG: Angiogenic effects of interleukin 8 (CXCL8) in human intestinal microvascular endothelial cells are mediated by CXCR2. J Biol Chem 278: 8508-8515, 2003.

36. Olson TS and Ley K: Chemokines and chemokine receptors in leukocyte trafficking. Am J Physiol Regul Integr Comp Physiol 283: R7-R28, 2002.

37. Hertzer KM, Donald GW and Hines OJ: CXCR2: A target for pancreatic cancer treatment? Expert Opin Ther Targets 17: 667-680, 2013.

38. Matsuo Y, Campbell PM, Brekken RA, Sung B, Ouellette MM, Fleming JB, Aggarwal BB, Der CJ and Guha S: K-Ras promotes angiogenesis mediated by immortalized human pancreatic epithelial cells through mitogen-activated protein kinase signaling pathways. Mol Cancer Res 7: 799-808, 2009.

39. Pan MR, Hsu MC, Luo CW, Chen LT, Shan YS and Hung WC: The histone methyltransferase G9a as a therapeutic target to override gemcitabine resistance in pancreatic cancer. Oncotarget 7: 61136-61151, 2016.

40. Matsuo Y, Sawai H, Ochi N, Yasuda A, Sakamoto M, Takahashi H, Funahashi H, Takeyama $\mathrm{H}$ and Guha S: Proteasome inhibitor MG132 inhibits angiogenesis in pancreatic cancer by blocking NF-kappaB activity. Dig Dis Sci 55: 1167-1176, 2010.

41. Qiao B, Luo W, Liu Y, Wang J, Liu C, Liu Z, Chen S, Gu J, $\mathrm{Qi} X$ and $\mathrm{Wu}$ T: The prognostic value of CXC chemokine receptor 2 (CXCR2) in cancers: A meta-analysis. Oncotarget 9: 15068-15076, 2017.

42. Song Y, Baba T, Li YY, Furukawa K, Tanabe Y, Matsugo S, Sasaki S and Mukaida N: Gemcitabine-induced CXCL8 expression counteracts its actions by inducing tumor neovascularization. Biochem Biophys Res Commun 458: 341-346, 2015.

43. Khan MA, Srivastava SK, Bhardwaj A, Singh S, Arora S, Zubair H, Carter JE and Singh AP: Gemcitabine triggers angiogenesis-promoting molecular signals in pancreatic cancer cells: Therapeutic implications. Oncotarget 6: 39140-39150, 2015.

44. Maliandi MV, Mato-Berciano A, Sobrevals L, Roué G, José A and Fillat C: AduPARE1 A and gemcitabine combined treatment trigger synergistic antitumor effects in pancreatic cancer through NF- $\mathrm{KB}$ mediated uPAR activation. Mol Cancer 14: 146, 2015. 